\title{
A new precise Co-geoid determined by spherical FFT for the Malaysian peninsula
}

\author{
M. N. J. P. Vella \\ Centre for Geodetic and Geodynamic Studies (CGGS), Faculty of Geoinformation Science and Engineering, \\ University of Technology Malaysia, Johor, Malaysia
}

(Received April 3, 2003; Revised June 30, 2003; Accepted July 14, 2003)

\begin{abstract}
A new gravimetric Co-geoid is produced for the Malaysian peninsula making it one of the most recent contributions to Co-geoid modelling in the region. Several data sources are included for the computations; these include 7447 terrestrial free-air gravity anomalies, 10243 altimeter derived gravity anomalies and 692881 mean digital elevation heights. The EGM96 global geopotential model to degree 40 is used in order to determine the long wavelength effect of the Co-geoid surface. Co-geoid undulations are determined through implementation of the remove-restore technique by combining the above-mentioned data sets with the geopotential model in a specific manner. The computations have been done using a two-dimensional spherical Fast Fourier Transform (FFT) with $100 \%$ zero padding. Terrain corrections are also computed using the FFT method and applied in the remove-restore technique. The absolute accuracy estimates are given for the Co-geoid undulations using 95 GPS control stations co-located with third order levelling. The comparisons show that using the Wong and Gore (1969) modification of Stoke's function for the long wave length effect using EGM96 up to degree/order 360 removed, and the modified Stoke's function with $L=40$ and a spherical cap radius of $1.6^{\circ}$, with data padding for areas of no gravity data produces a Co-geoid which gives a bias of 0.378 meters and a standard deviation of \pm 0.277 meters respectively. Where no data padding is used a Co-geoid is produced which gives a bias of 0.439 meters and a standard deviation of \pm 0.284 meters respectively, thus showing the improvement of the Co-geoid solution through the data padding.
\end{abstract}

Key words: Co-geoid, GTOPO30, KMS01, data padding.

\section{Introduction}

With the advent of the Global Positioning System (GPS) and techniques that allow improved and increased accurate measurements of geodetic parameters from GPS (see Shaowei and Rizos, 1996), the determination of the Co-geoid has become of prime importance for many in the geodetic and geophysical communities. GPS provides the framework for the very accurate determination of three dimensional cartesian coordinates $X, Y, Z$, which are easily converted to latitude $(\phi)$, longitude $(\lambda)$ and ellipsoidal height $(h)$. Latitude and longitude define planimetric position in two dimensions, but in order to obtain a practical three-dimensional system the ellipsoidal height needs to be converted into an orthometric height, which involves combining the ellipsoidal height and the Co-geoid height. This combination gives rise to the determination of the Co-geoid in that the Co-geoid undulation is used to provide GPS users with accurate relative orthometric heights via the Global Positioning System. With the level of precision and accuracy now obtainable for ellipsoidal heights the same level of accuracy in the gravimetric Co-geoid heights should also be aimed at (e.g., Schwarz et al., 1987).

Through the combination of relative ellipsoidal heights $\Delta h$ from GPS and relative Co-geoid heights $\Delta N$, orthometric height differences $\Delta H$ can be determined by the equa-

Copy right(C) The Society of Geomagnetism and Earth, Planetary and Space Sciences (SGEPSS); The Seismological Society of Japan; The Volcanological Society of Japan; The Geodetic Society of Japan; The Japanese Society for Planetary Sciences. tion: $\Delta H=\Delta h-\Delta N$. This indicates that the accuracy of all variables should be approximately the same. In order to understand fully the level of accuracy of the terms of the above equation, a proper error analysis should be undertaken as described in Kotsakis and Sideris (1999). This provides a detailed treatment of the adjustment problem taking into account the affect on the orthometric height by presenting a pure deterministic parametric model, and a hybrid deterministic and stochastic model. This has not been attempted here due to the lack of a priori information for the ellipsoidal heights and other geodetic parameters.

Previously in the Malaysian peninsula, Kadir et al. (1999) has shown that EGM96 (Lemoine et al., 1996) is the optimal reference field, for the Malaysian peninsula region. This latest Co-geoid is also making use of EGM96, which is used in the remove-restore technique for the gravimetric Cogeoid computations. It is also shown by Lemoine et al. (1998), that EGM96 was improved in many parts of the globe by the inclusion of extra data with specific attention to South East Asia. This study presents the most current attempt to compute the Co-geoid in the Malaysian Peninsula, including the most recently available data, such as EGM96 the long wavelength reference model, KMS01 a globally available set of gravity anomalies developed by Andersen and Knudsen (1998), from ERS1 and GEOSAT geodetic mission altimetry, point terrestrial free-air gravity anomalies and height data in the form of a mean 30 second height DEM GTOPO30. By applying the remove-restore technique 
Table 1. Differences for interpolated heights from the DEM and heights from 7447 gravity stations.

\begin{tabular}{ccccc}
\hline & Mean $(\mathrm{m})$ & $\pm \sigma(\mathrm{m})$ & Min $(\mathrm{m})$ & Max $(\mathrm{m})$ \\
\hline GTOPO30 - Station heights $(30 \mathrm{sec})$ & -32.941 & 62.645 & -1106.872 & 181.162 \\
GTOPO30 - Station heights $(1 \mathrm{~min})$ & -34.326 & 63.380 & -1111.536 & 217.729 \\
\hline
\end{tabular}

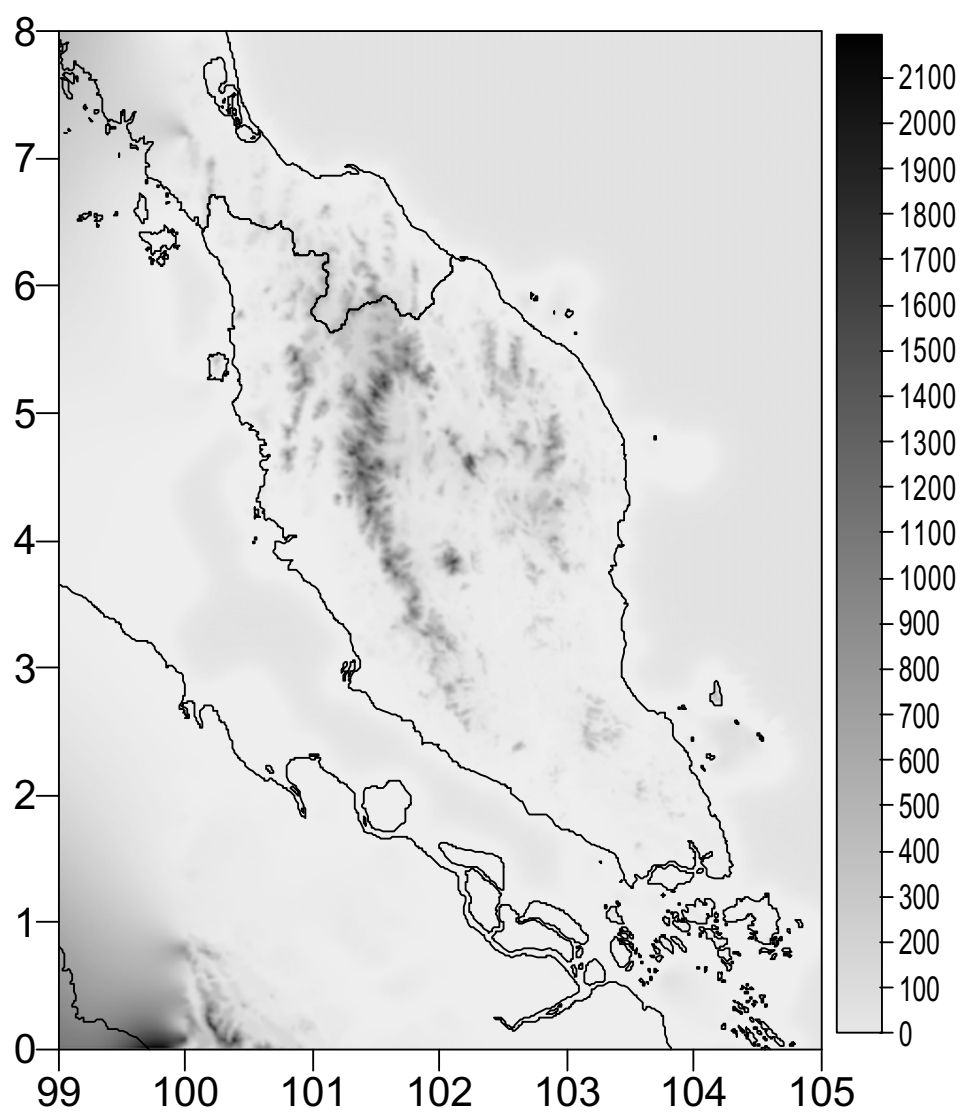

Fig. 1. GTOPO30 digital elevation model (mean $30 \mathrm{sec}$ grid spacing).

new improved Co-geoid undulations are computed through the combination of the EGM96 model, the terrestrial gravity data, the altimeter data and the heights. The new set of Co-geoid undulations are based on Stokes' integration of gridded gravity through the spherical FFT method with the inclusion of terrain corrections but not accounting for the indirect effect, thus the terminology Co-geoid. The software package GRAVSOFT has been used in this study.

\section{Data Sets Used \\ 2.1 GTOPO30 DEM}

GTOPO30 is a global Digital Elevation Model (DEM) resulting from a collaborative effort led by the U.S. Geological Survey's EROS Data Centre. The mean elevations in GTOPO30 are regularly spaced at 30-arc seconds (approximately 1 kilometre). GTOPO30 was developed to meet the needs of the geospatial data user community for regional and continental scale topographic data.

A comparison between the heights from the gravity stations and the corresponding mean heights from the DEM is made. This is done in order to see how well the DEM represents the topography of the Malaysian Peninsula. The heights from the DEM will be used to transform the grid- ded Bouguer anomalies back to free-air, for use in the Cogeoid computations. Table 1 represents the statistics of the comparisons, whereby the gravity station heights are interpolated from the mean height of the DEM and compared accordingly. The grid spacing chosen to do the Co-geoid computations is 1 minute, therefore the DEM is also re-sampled at 1 minute, for which the comparisons are shown.

The mean height from the DEM is not capable of recovering accurate heights. It should be remembered that the comparisons are conducted over terrain which has rather steep gradients and is changing rapidly making it hard for a mean $1 \mathrm{~km}$ spaced DEM to give heights corresponding to these changes. It is apparent from Table 1 , that there is a bias of around $-32 \mathrm{~m}$ between the DEM and the local vertical datum. This could introduce errors into the terrain corrections as well as the free-air anomalies which result from the DEM. The bias exists due to the fact of the great deviations in gradient of the undulating terrain when point values are compared to mean values. The bias is not modelled in this case, however it is suggested that in order to verify the accuracy of the DEM to the Malaysian terrain, at least a local 10 second DEM consisting of mean heights should be used, at this time there is no such local DEM available for these tests. Figure 1 


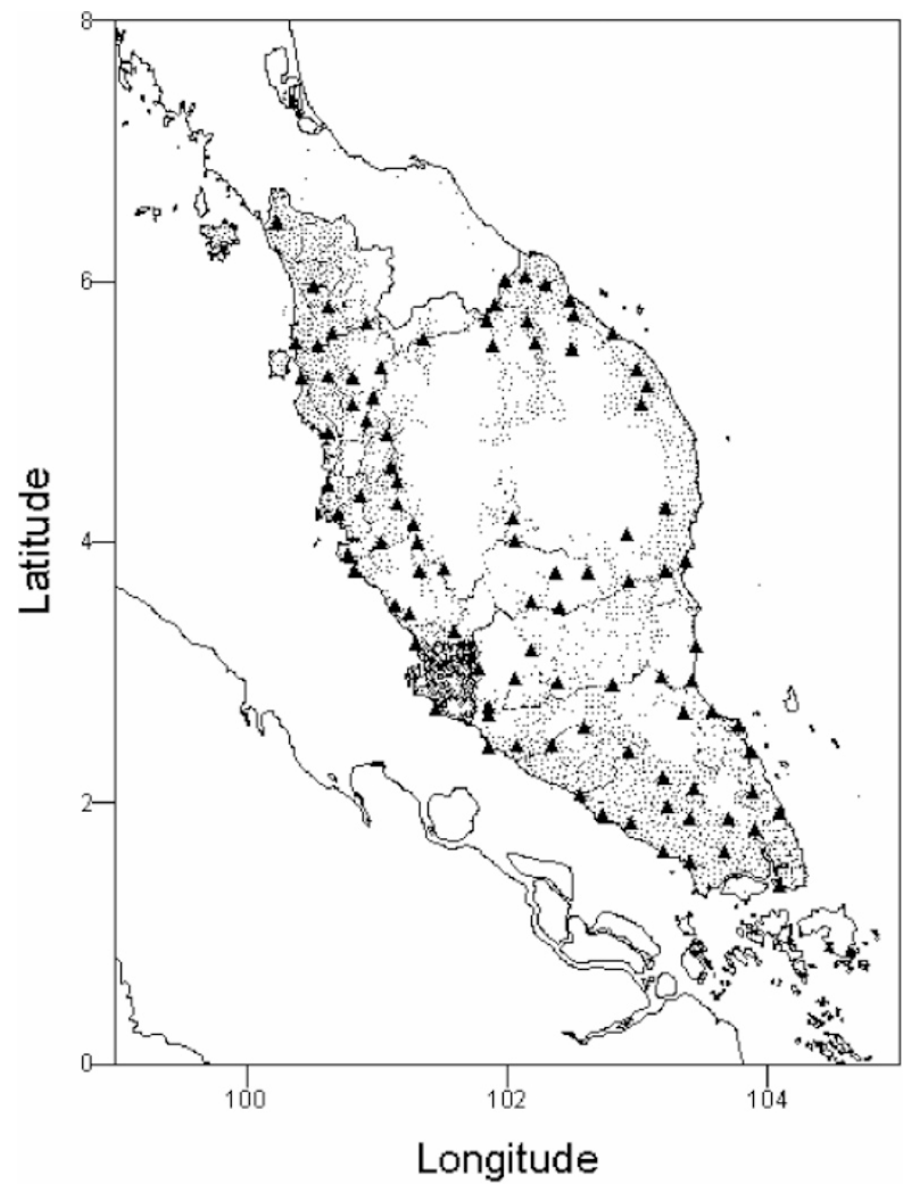

Fig. 2. The location of the Malaysian peninsula terrestrial gravity data, with GPS control stations (indicated by triangles) used in this study.

shows GTOPO30 in its original 30 second form.

\subsection{KMS01 marine altimeter model}

There are generally four methods used for observing the gravity field of the marine environment. These methods are 1) Gravimeters placed on board vessels, 2) Gravity meters lowered to the ocean floor (used rarely) 3) Airborne platforms such as Airborne Gravimetry and 4) The sea surface height derived from satellite altimeters. Satellite altimetry is fundamentally different from the first three because the observations of the sea surface height are used to infer the gravity field (Andersen and Knudsen, 2000).

KMS01 is an improvement upon KMS98 and KMS99 a globally available set of gravity anomalies developed by Andersen and Knudsen (1998) from ERS1 and GEOSAT geodetic mission altimetry. The ERS1 altimetry mission covers all oceans between latitudes $82^{\circ} \mathrm{S}$ and $82^{\circ} \mathrm{N}$ and provides a dense $\left(2^{\prime}\right.$ by $\left.2^{\prime}\right)$ homogeneous coverage. The GEOSAT geodetic mission contains data from the first 18 months of the mission yielding a very dense though not completely homogeneous coverage of satellite altimeter observations. The ERS1 and GEOSAT satellite altimeter missions provide geodesists with the chance to make very detailed mappings of the marine gravity field (Andersen and Knudsen, 2000). Andersen and Knudsen (2000) used a combination of FFT and collocation techniques to derive the global model, which is spaced at $2^{\prime}$ by $2^{\prime}$ globally. For a thorough discussion on the methods used and the data types used the reader is referred to Andersen and Knudsen (2000).

\subsection{Terrestrial point gravity data}

Currently Malaysia has an existing gravity data base of 7447 points, Figure 2 shows the coverage and distribution of the free-air anomaly for Peninsula Malaysia. The International Gravity Standardisation Net, 1971 (IGSN71) stations at Kuala Lumpur and Singapore provide the scale and the datum of the base net (Kadir and Ses, 1989). Due to the mountainous regions in the interior of the country it is virtually financially impossible to mount sustainable campaigns for the collection of gravity data. This will shortly be solved as The Department of Survey and Mapping Malaysia (JUPEM) are due to undertake airborne gravimetric missions, which will infill almost all of the areas, which are considered unreachable by terrestrial methods.

\subsection{Augmentation of data sets using data padding}

The procedure described here is not new, but it is unique and what makes it so is the freely available global mean DEM, which provides National Survey bodies access to relatively precise approximations of the terrain while in the interim developing their own more accurate DEM.

The correlation between the free-air anomaly and its corresponding height can be expressed approximately in linear form as such:

$$
\Delta g_{F}=a+b \cdot h
$$

where $a$ and $b$ are the intercept and regression coefficient, respectively. These quantities can be derived using least squares regression. 


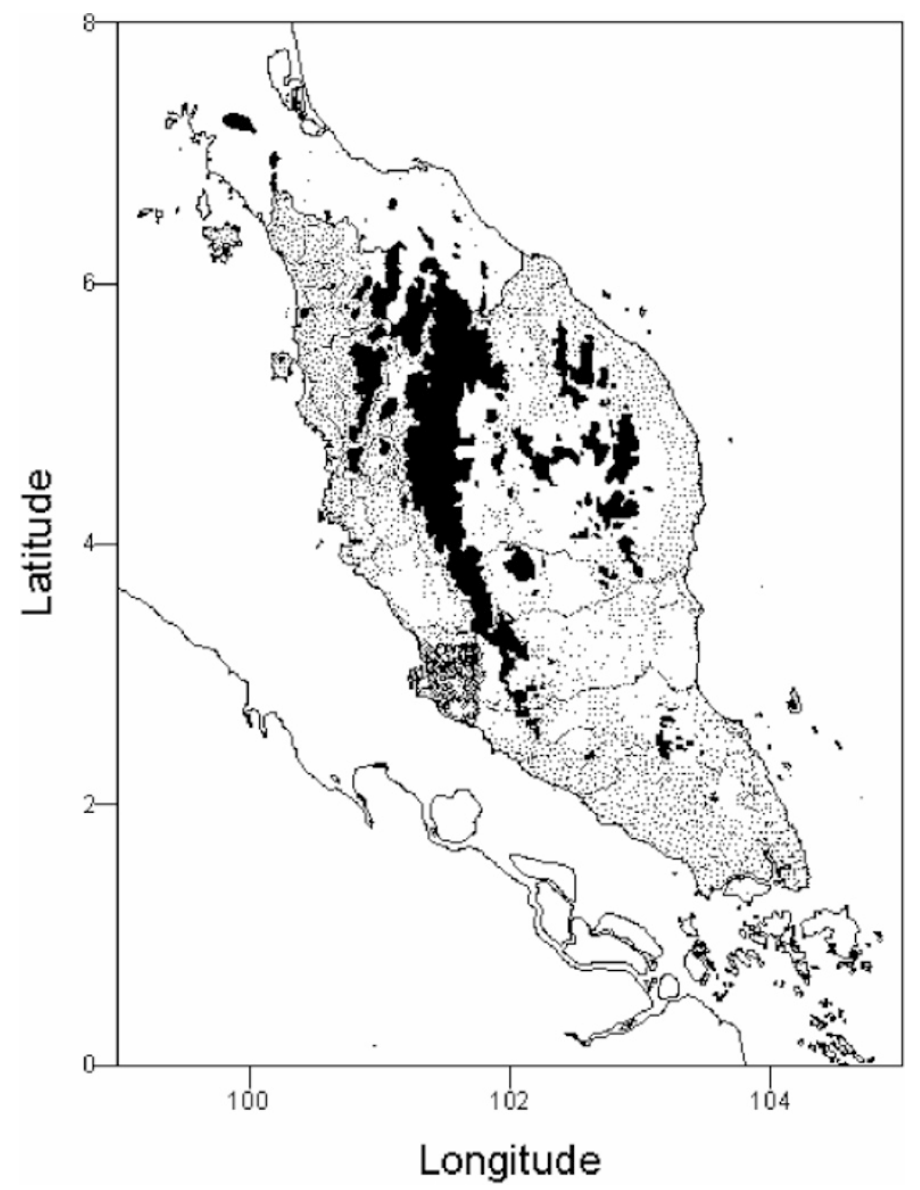

Fig. 3. Augmentation of the existing gravity data with the modelled data used in the data padding process.

As mentioned least squares regression is used in the current study with orthometric height $(H)$ taken from observed heights recorded in the observing phase of the gravity data. Tests are conducted with a total of 6 sets of minimum heights chosen as cut off points for the derivation of the correlation coefficient. This was done in order to determine the optimum height when modelling the correlation between the height of the observed gravity data and the free-air anomaly.

Including data with heights less than $400 \mathrm{~m}$ decreases the correlation coefficient, whereas $400 \mathrm{~m}$ is considered the optimum lower height cut off, not only because of the correlation coefficient but also because of the number of data and the distribution of these data. This inclusion of the modelled data prior to gridding in effect helps to control gridding of the data to be used as input into the FFT Co-geoid computations. Using the data set not containing the modelled correlated data for gridding leaves vast gaps, thus introducing errors in the interpolation by the gridding algorithms used. By including the modelled data taken from the DEM with a height of not less than $400 \mathrm{~m}$ ensures adequate coverage and spacing of data for input into the gridding procedure. Figure 3 shows the existing gravity data base with the augmented modelled data used in the data padding procedure.

\subsection{GPS/levelling data}

The Department of Surveying and Mapping Malaysia (JUPEM), provided the GPS control stations, in all a total of 230 stations. However, due to the rugged terrain of the Malaysian peninsula's interior not all GPS stations are co-located with orthometric heights. Some heights are trigonometrically levelled and others are barometrically derived. For the comparisons only those heights, which are truly orthometric heights, are considered and after screening give a total of 95 points. Figure 2 also shows the distribution of the GPS control stations used in this study, with respect to the observed gravity data.

\section{Gridding and Co-geoid Computations}

Data used in the Co-geoid computations are grouped together so that the relevant steps for optimum computation of the Co-geoid can be undertaken. The optimum computation methodology is derived from comparing different cap sizes for the spherical cap integration. The choice of the cap size is done by way of comparing each individual Co-geoid solution with the 95 GPS points, the solution with the lowest standard deviation of the differences is chosen. Tests were done for the cap size integration radius; these results are discussed later on. Before any gridding is done, the free-air data $\Delta g_{F}$ provided by JUPEM, has EGM96 removed, as the initial step in the remove-restore technique. The procedure here follows a similar procedure as that of Vella and Featherstone (1999), however different software is used and only one kernel modification is considered, without the indirect effect applied. Also the Bouguer anomaly is gridded here, not the free-air anomaly. The altimetry data also has EGM96 removed and is combined with the terrestrial gravity data. This combined residual data set is then transformed into Bouguer anomalies 


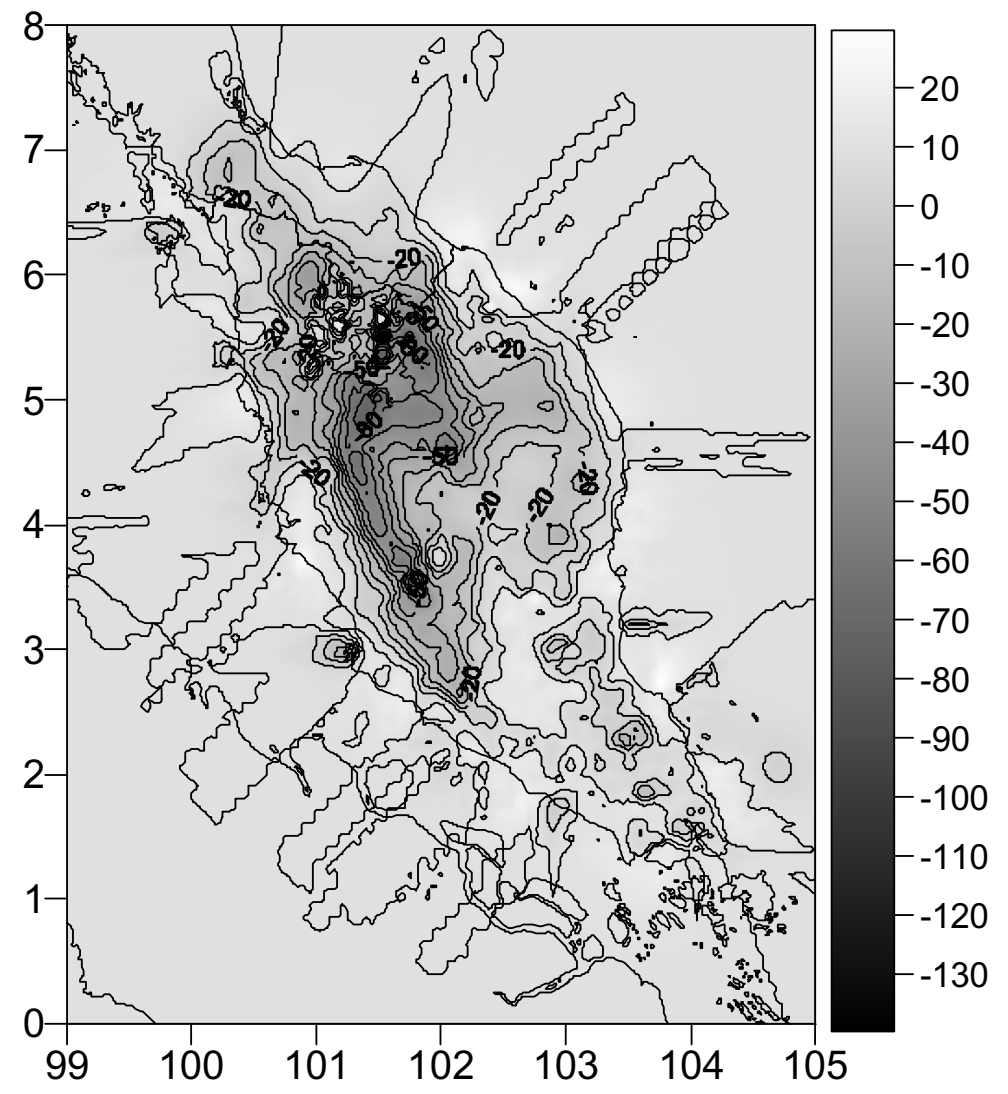

Fig. 4. Residual Bouguer anomalies gridded at an interval of 1 minute, units in mGals. Contour Interval $10 \mathrm{mGals.}$

Table 2. Statistics for the different gravity data sets used in the computations, all units in mGals. ( $\Delta g_{B}$ is the Bouguer anomaly, $\Delta g_{B}{ }^{r e s}$ is the residual Bouguer anomaly (i.e.: EGM96 removed), KMS01 is the gravity derived from the altimeter model, KMSO1_rd is the gravity derived from the altimeter model reduced, $\Delta g_{\bar{F} \text { res }}$ is the mean residual free-air anomaly).

\begin{tabular}{lcrrrr}
\hline & No of Data & Mean & \multicolumn{1}{c}{$\pm \sigma$} & \multicolumn{1}{c}{ Min } & \multicolumn{1}{c}{ Max } \\
\hline$\Delta g_{B}$ & 7447 & 8.069 & 17.344 & -70.329 & 96.929 \\
$\Delta g_{B^{r e s}}$ & 7447 & -14.072 & 19.478 & -105.328 & 73.956 \\
KMS01 & 10243 & 12.233 & 13.713 & -37.750 & 107.900 \\
KMS01_rd & 10243 & 0.374 & 6.492 & -55.405 & 36.476 \\
$\Delta g_{\bar{F} \text { res }}$ & 173641 & 12.570 & 24.040 & -63.010 & 248.560 \\
\hline
\end{tabular}

by applying the Bouguer reduction in the form of:

$$
\Delta g_{B}=\Delta g_{F}-A_{B}
$$

where: $\Delta g_{B}$ is the complete Bouguer reduced anomaly referring to the Co-geoid, $\Delta g_{F}$ is the free-air anomaly and $A_{B}$ is the correction for the attraction of an infinite Bouguer plate (Heiskanen and Moritz, 1967, pp. 130-131). With standard density $\rho=2.67 \mathrm{~g} / \mathrm{cm}^{3}$ this becomes $A_{B}=0.1119 h$, where $h$ is the height in meters.

The assumption that terrain corrections should not be applied in the marine region is assumed plausible considering the coastal areas of the Malaysian peninsula are low, flat areas, in any case the inland terrain itself is not that demanding in terms of correcting for terrain, statistics for the terrain correction are presented in a later section. The marine area of the study is well catered for by way of the KMS01 altimeter gravity model, however the existing free-air terrestrial anomaly data set is still somewhat lacking. It is envisaged that future planned campaigns of airborne gravimetric sur- veys will solve this problem and augment the existing data set. A supplementary set of mean free-air anomalies is derived based on the mean DEM GTOPO30 and the fact that gravity is highly correlated to height. The highest correlation was found when using a lower height cut off of 400 meters, which was well suited to the areas where there were no data in the middle of the Peninsula, see Section 2.4. This set of mean anomalies was combined with the data from JUPEM at the initial stage before removal of EGM96, this augmented data has the effect of reducing the overall bias between the Co-geoid and the existing height datum.

The residual Bouguer anomaly (i.e: with EGM96 removed to degree/order 360 ) given by $\Delta g_{B^{r e s}}$ is used in the gridding. Figure 4 shows the gridded residual Bouguer anomalies, gridded at one-minute interval, and Table 2 shows the statistics of some of the data sets used.

The residual Bouguer anomaly $\Delta g_{B^{r e s}}$ is used as the Bouguer anomaly itself is considered smoother than the freeair anomaly and less prone to aliasing or pseudo-aliasing as 


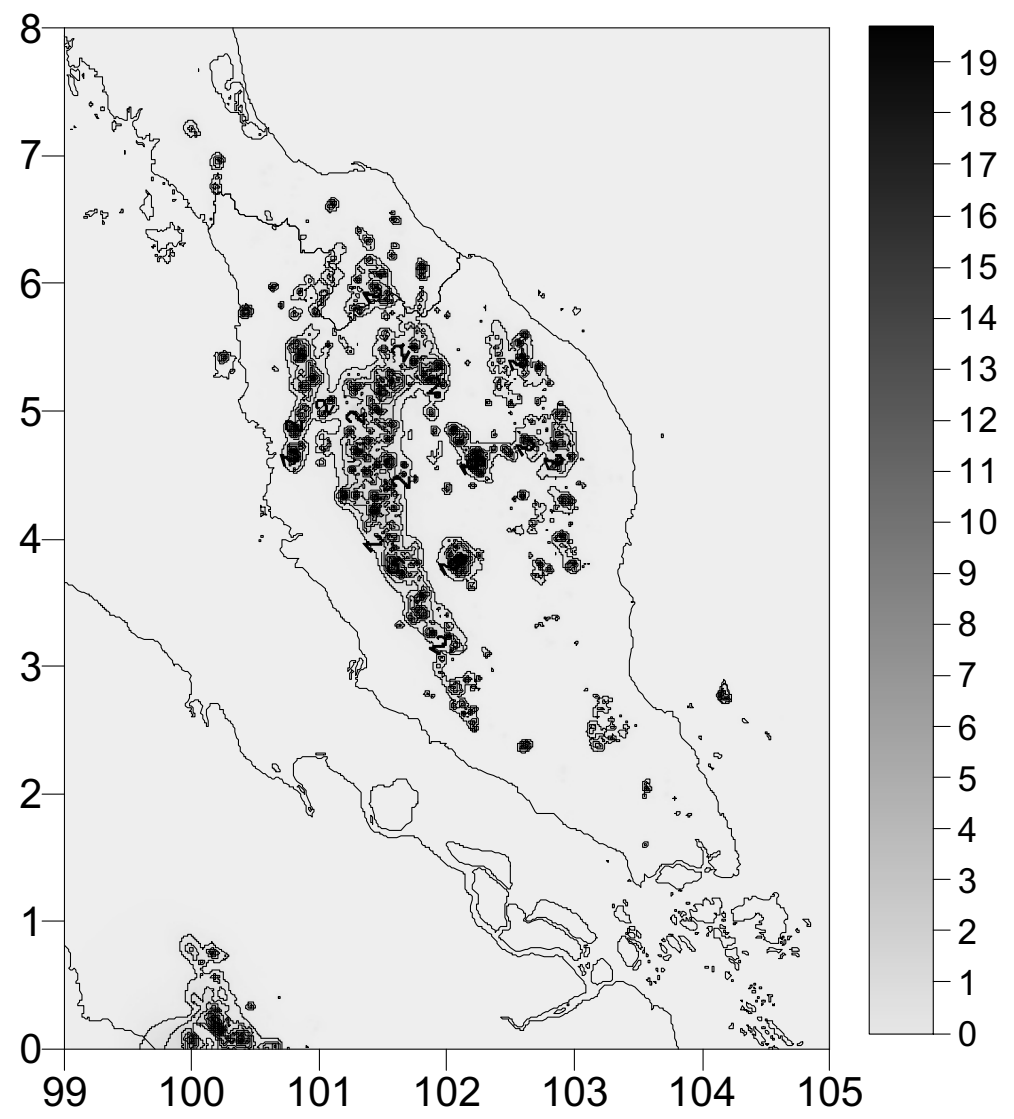

Fig. 5. Terrain corrections computed from GTOPO30, resampled at 1 minute. Contour Interval $1 \mathrm{mGal.}$

described in Goos et al. (in press). The Bouguer anomaly $\Delta g_{B}$ has EGM96 removed in order to remove the long wavelength effect of the Earths geopotential field:

$$
\Delta g_{B^{r e s}}=\Delta g_{B}-\Delta g_{\mathrm{EGM} 96}
$$

This residual set of Bouguer anomalies is then gridded at an interval of 1 minute and interpolated onto a regular grid using Eq. (2), providing regularly gridded residual Bouguer anomalies. The residual Bouguer anomalies are then used together with the mean DEM GTOPO30, in order for the residual mean free-air anomalies $\left(\Delta g_{\overline{F r e s}}\right)$ to be derived:

$$
\Delta g_{\overline{F r e s}}=\Delta g_{B^{r e s}}+2 \pi G \rho H
$$

where $2 \pi G \rho$ is approximated by 0.1119 and $H$ is the height interpolated from the DEM.

Collocation or krieging is used for the gridding; a secondorder isotropic Markov function is used such that:

$$
c(r)=C_{0}\left(1+\frac{r}{\alpha}\right) e^{\left(\frac{-r}{\alpha}\right)}
$$

where: $r$ is the spatial distance, $C_{0}$ is the error variance (determined automatically) and $\alpha$ is the correlation length $(25 \mathrm{~km})$.

The Co-geoid height $\left(\mathrm{N}^{\mathrm{c}}\right)$ is the estimate of the separation between the Co-geoid and the reference ellipsoid, which can be found in the following Heiskanen and Moritz, (1967), p. 95.

In order to see in which way the FFT can be applied to Stokes's formula utilizing an exact number of bands, refer to Forsberg and Sideris (1993).
In all computations the terrain corrections have been included at the final stage of the computation process before being operated on by the FFT.

\subsection{Wong and Gore kernel modification}

A modified integration kernel is produced when the low degree Legendre polynomials are removed from the spherical Stokes integration kernel, this is what is suggested by Wong and Gore (1969) and has been incorporated into software for Co-geoid computations.

\subsection{Terrain corrections}

The approximation used represents the 'linear approximation' to the terrain correction Schwarz et al. (1990) and is given also in Moritz (1968).

In the DEM, all heights less than zero are set to zero in order that no terrain correction is added to the marine area, as previously mentioned. The terrain corrections are applied to the transformed residual mean free-air anomalies $\left(\Delta g_{\bar{F} r e s}\right)$, which in order provide terrain corrected residual mean freeair anomalies.

Figure 5 shows the terrain correction computed from GTOPO30, which is re-sampled at 1 minute, Table 3 shows different grid sizes for the terrain corrections. The standard deviations reduce with large grids and so does the maximum value.

\section{Results and Conclusion}

In order to determine the most suitable spherical cap size, several tests were carried out where all data sets were chosen on a grid interval of 1 minute, Figure 6 shows the results 
Table 3. Terrain corrections computed from GTOPO30, on $1^{\prime}, 2^{\prime}$ and $3^{\prime}$ grid intervals.

\begin{tabular}{lccc}
\hline & Mean & $\pm \sigma$ & Max \\
\hline Terrain corrections $(1 \mathrm{~min}) \mathrm{mGals}$ & 0.250 & 0.790 & 19.700 \\
Terrain corrections $(2 \mathrm{~min}) \mathrm{mGals}$ & 0.250 & 0.800 & 18.110 \\
Terrain corrections $(3 \mathrm{~min}) \mathrm{mGals}$ & 0.210 & 0.650 & 13.320 \\
\hline
\end{tabular}

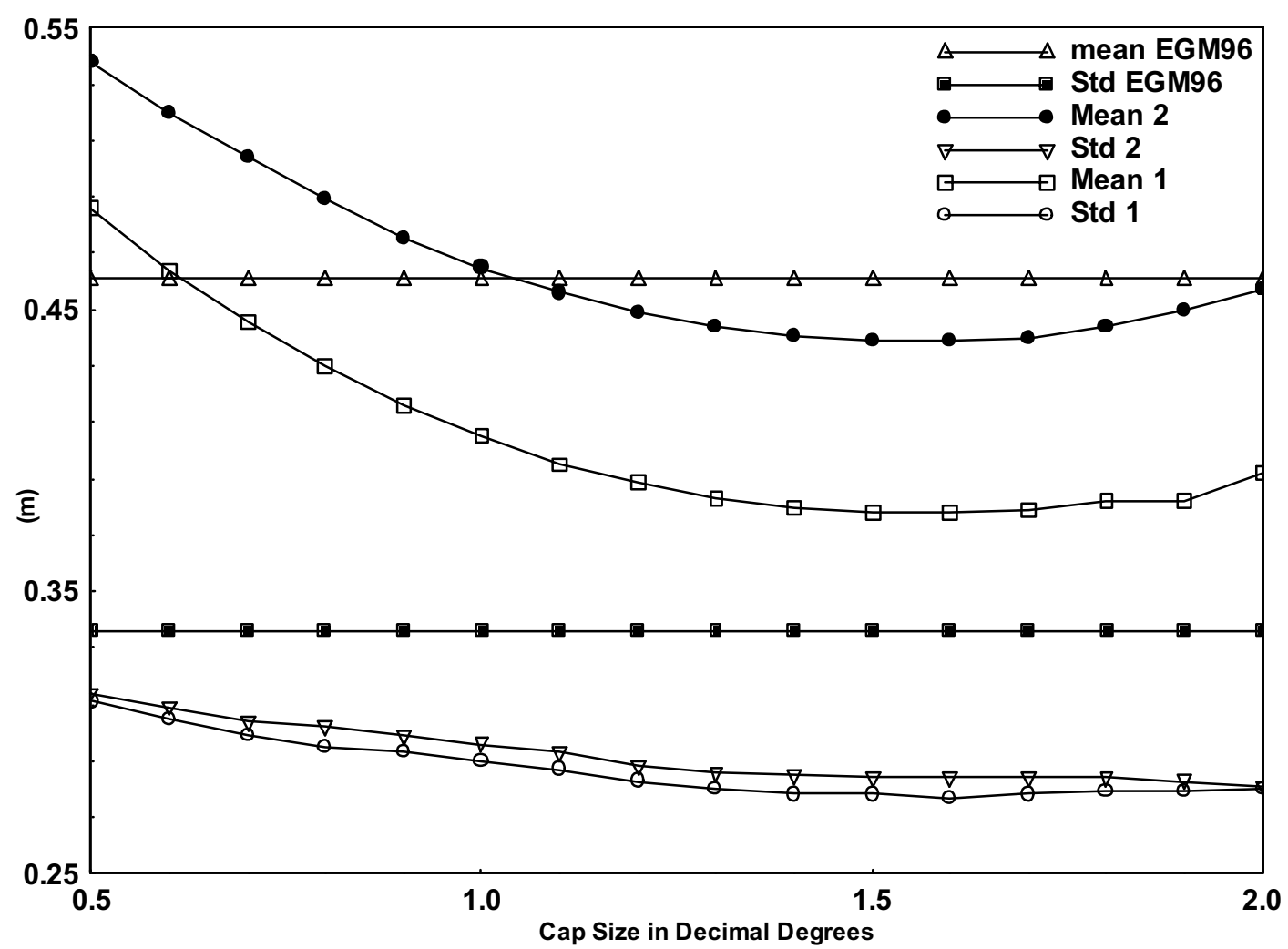

Fig. 6. Test showing the difference between $\mathrm{N}_{\mathrm{GPS}}$ and $\mathrm{N}_{\mathrm{Co} \text {-geoid }}$ for different spherical cap sizes, also EGM96 is represented by the solid constant lines in standard deviation and mean.

from the comparison with GPS points, where the data derived using the correlation technique is augmented as data padding. In Fig. 6 the spherical cap radius was chosen from 2 degrees to 0.5 degrees decreasing at 0.1 degree. This can be seen on the horizontal axis of Fig. 6, with the vertical axis showing the result of the differences between the Co-geoid solution and the GPS points. Plotted together with the standard deviation is the mean, this is done in order to demonstrate how the varying cap size also affects the bias between the vertical datum and the Co-geoid. By way of choosing an appropriately limited cap size it is also possible to improve the bias. In this case the bias was improved from 0.486 meters at 0.5 degrees cap radius to 0.378 meters at 1.6 degrees cap radius. Figure 6 , shows the comparisons combined in order to visualise the improvement in the overall bias due to the data padding. This clearly indicates the advantage of using the data padding for gridding purposes over not using it. Table 4, shows the results for the comparisons for a cap size of 1.6 degrees, the optimum cap size as shown in this study. The mean (bias) has been reduced by more than 0.05 meters, the reasons for this bias are as mentioned in Kotsakis and Sideris (1999). These might be, (1) random noise in the ellipsoidal heights, orthometric heights or geoid/ellipsoid sep-
Table 4. Test showing the difference between $\mathrm{N}_{\mathrm{GPS}}$ and $\mathrm{N}_{\text {Co-geoid }}$ for a spherical cap size of 1.6 degrees.

\begin{tabular}{lc}
\hline & 1.6 \\
\hline$\sigma_{1}$ & 0.277 \\
$\bar{x}_{1}$ & 0.378 \\
$\sigma_{2}$ & 0.284 \\
$\bar{x}_{2}$ & 0.439 \\
$\sigma_{\text {EGM96 }}$ & 0.336 \\
$\bar{x}_{\text {EGM96 }}$ & 0.461 \\
\hline
\end{tabular}

arations, (2) datum inconsistencies and other systematic distortions, (3) various geodynamic effects and (4) theoretical approximations in the computation of either the orthometric height $(\mathrm{H})$ or the geoid/ellipsoid separation $(\mathrm{N})$ are not good enough. Peninsular Malaysia lies well away from any major subduction zones, the possible reasons for (3) could well be land subsidence at tide gauges used for the vertical datum and or mean sea level rise. However these reasons were not researched in this current paper.

Figure 6, demonstrates that a spherical cap size of 1.6 de- 


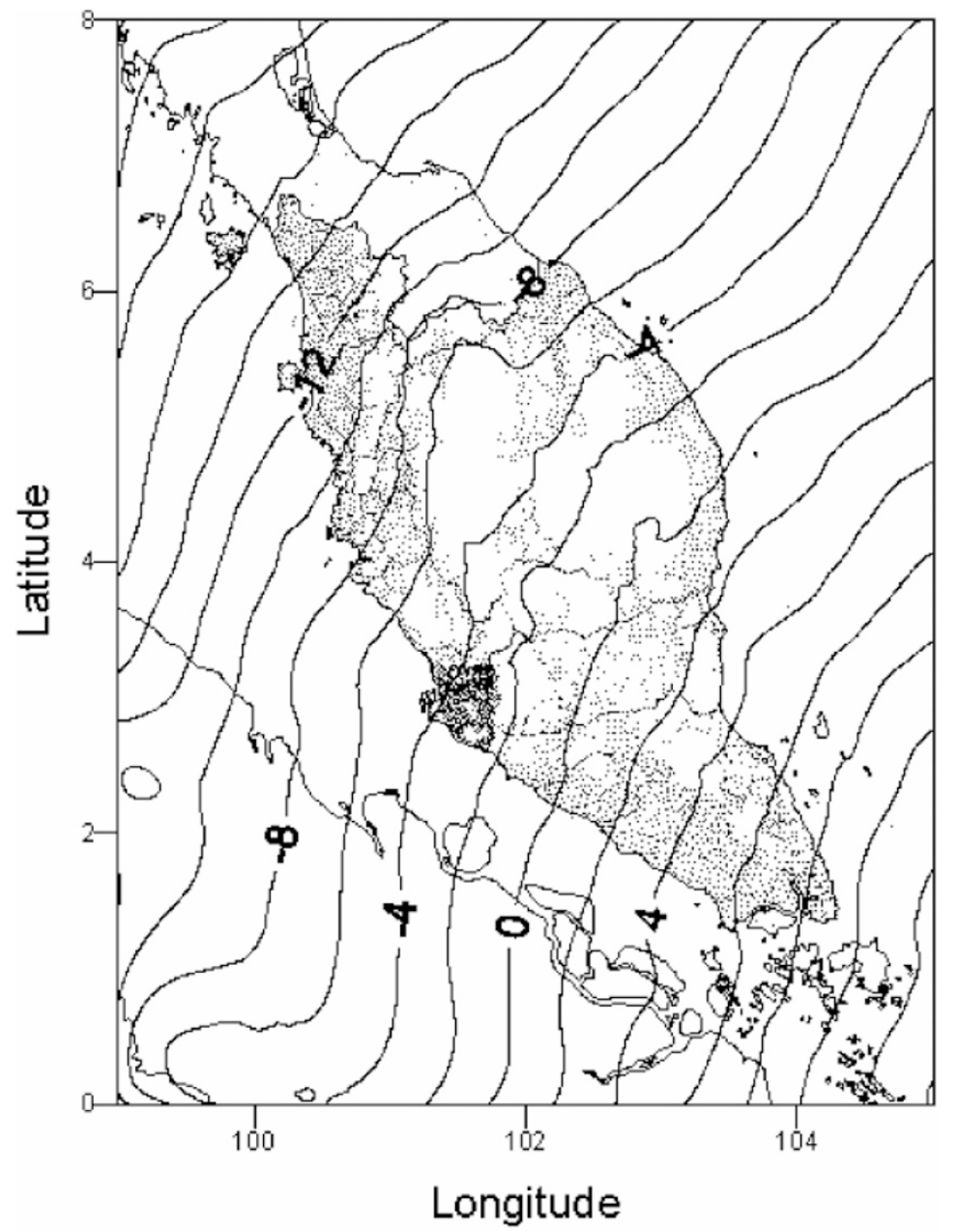

Fig. 7. Resulting Co-geoid (data padding) map, with EGM96 restored. Based on the Wong and Gore kernel modification with a spherical cap size of 1.6 degrees. Contour Interval $2 \mathrm{~m}$.

grees gives the smallest standard deviation. This agrees with the assertion made by Heck and Gruninger (1988), that the first zero-crossing (located at approximately 1.6 degrees with Wong-Gore modified kernel with $L=40$ ) should be used in the calculation of the Co-geoid. Also shown in Fig. 6 are the results from comparisons of the 95 GPS stations to EGM96, it is evident that the new Co-geoid is clearly an improvement on the global long wavelength model EGM96. The final Cogeoid (data padding) is shown in Fig. 7, where it is shown the effect of adding back the removed long wavelength effect from EGM96.

By comparing the GPS points and ignoring the bias, i.e.: the mean, it is possible to consider the standard deviation of the residuals and emphasise how much better the Co-geoid with the limited cap size is. The spurious effects seen in the south western corner are due to the contribution of the DEM over Indonesia. No gravity data was available for these computations from Indonesia and it is therefore suggested that Co-geoid undulations in this particular small region are to be considered unreliable. Also if the distribution of the gravity data is considered, i.e. Figure 2, then the Co-geoid values for the region in the central highlands of the Malaysian peninsula would be considered to be not truly representative as there is a great lack of coverage in this region. Also, as Figure 2 suggests, the distribution of the GPS control points does not allow for a thorough examination of the ability of the Co-geoid model to recover the Co-geoid heights.

Overall the Co-geoid that includes augmented data padding shows an improvement in the Co-geoid solution for the Malaysian Peninsula, with respect to EGM96 and the associated 95 GPS/Levelling stations.

Acknowledgments. The Author would like to thank Rene Forsberg of the KMS for his lengthy discussion and comments on this paper and for his suggestions in the write up. Also thanks to Dr. Teng Chee Hua, Director of Geodesy, JUPEM for providing the gravity and GPS data. This research is supported by a short term research grant vot number 71922, under the University of Technology Malaysia. Also the comments of the two reviewers are very much welcomed and appreciated.

\section{References}

Andersen, O. B. and P. Knudsen, Global marine gravity field from the ERS1 and GEGOSAT geodetic mission altimetry, Journal of Geophysical Research, 103(C4), April 15, 1998.

Andersen, O. B. and P. Knudsen, The role of satellite altimetry in gravity field modelling in coastal areas, Phys. Chem. Earth (A), 25(1), 17-24, 2000.

Forsberg, R. and M. G. Sideris, Co-geoid computations by the multiband spherical FFT approach, Manuscripta. Geodaetica., 18, 82-90, 1993.

Goos, J. M., W. E. Featherstone, S. A. Holmes, and J. F. Kirby, Experiments with two different approaches to gridding terrestrial gravity anomalies and their effect on regional Co-geoid computation, Survey Review, 2003 
(in press).

Heck, B. and W. Gruninger, Modification of Stokes' integral formula by combining two classical approaches. In I. U of Geodesy and V. Geophysics, edited by XiX General Assembly, Proc IAG Symp, volume 2, pp. 319-337, Paris, Bureau central de l'A.L.G., 1988.

Heiskanen, W. A. and H. Moritz, Physical Geodesy, 364 pp, W. H. Freeman and Co., San Francisco, USA, 1967.

Kadir, M. and S. Ses, Towards the development of a national gravity database, The Surveyor, The Professional Journal of the Institution of Surveyors Malaysia, 24(4), 1989.

Kadir, A. M. A., H. Fashir, and K. Omar, A regional gravimetric Co-geoid over South East Asia, Geomatics Research Australasia, 71, June, 37-56 1999.

Kotsakis, C. and M. G. Sideris, On the adjustment of combined GPS/levelling/Co-geoid networks, Journal of Geodesy, 73, 412-421, 1999.

Lemoine, F. G., S. C. Kenyon, J. K. Factor, R. G. Trimmer, N. K. Pavlis, D. S. Chinn, C. M. Cox, S. M. Klosko, S. B. Luthcke, M. H. Torrence, Y. M. Wang, R. G. Williamson, E. C. Pavlis, R. H. Rapp, and T. R. Olson, The Development of the Joint NASA GSFC and NIMA Geopotential Model EGM96, NASA Goddard Space Flight Center, Greenbelt, Mary- land, 20771 USA, NASA/TP-1998-206861, July 1998.

Moritz, H., On the use of the terrain correction in solving Molodensky's problem, Report 108, Department of Geodetic Science and Surveying, The Ohio State University, Columbus, USA, 1968.

Schwarz, K.-P., M. G. Sideris, and R. Forsberg, Orthometric heights without levelling, J. Surv. Eng., 113(1), 28-40, 1987.

Schwarz, K. P., M. G. Sideris, and R. Forsberg, The use of the FFT techniques in physical geodesy, Geophysical Journal International, 100, 485514, 1990.

Shaowei, H. and C. Rizos, Improving the computational efficiency of the ambiguity function algorithm, Journal of Geodesy, 70, 330-341, 1996.

Vella, J. P. and W. E. Featherstone, A gravimetric Co-geoid model of Tasmania, computed using the one-dimensional Fast Fourier Transform and a deterministically modified kernel, Geomatics Research Australasia, 70, 53-76, June 1999.

Wong, L. and R. Gore, Accuracy of Co-geoid heights from modified Stokes kernels, Geophysical Journal of the Royal Astronomical Society, 18, 8191, 1969.

M. N. J. P. Vella (e-mail: nur_jason@fksg.utm.my) 